\title{
Related Factors Associated with Exclusive Breastfeeding in Isfahan Province
}

\section{A R T I C L E I N F O}

\section{Article Type}

Original research

\section{Authors}

Javanmardi M. ${ }^{1} M S C$

Khodakarami N.* $M S c$,

Jannesari Sh. ${ }^{1} M S c$,

Akbarzadeh Baghban A.R. ${ }^{2}$ PhD

\section{A B S T R A C T}

Aims Exclusive breastfeeding plays an important role in the growth and promotion of children's health in the first six months of their life. The exclusive breastfeeding has been decreasing in Iran in recent years. The objective of this study was to investigate the factors associated with the exclusive breastfeeding in Isfahan province, Iran.

Materials \& Methods This cross-sectional study was conducted on women with infants less than 6 months of age who referred to the urban and rural health centers of Golpayegan, Isfahan and Falavarjan counties (561 people) of Isfahan province between 2007 and 2008. The data were collected using a questionnaire and interview. Statistical analyses were performed using SPSS 15 software and logistic regression model.

Findings Most of the infants (85.6\%) fed colostrum. The exclusive breastfeeding levels in the urban and rural regions were found as $25.9 \%$ and $32.4 \%$, respectively. Delayed onset of lactation $(\mathrm{p}<0.05)$ and cesarean section $(\mathrm{p}<0.05)$ were associated with reduction in exclusive breastfeeding, while having a female gender $(\mathrm{p}<0.01)$ and receiving postpartum lactation training $(\mathrm{p}<0.001)$ showed a positive association with exclusive breastfeeding.

Conclusion Delayed onset of lactation and cesarean section reduce the level of exclusive breastfeeding in infants less than 6 months of age in urban and rural communities of Isfahan. However, having a female gender and receiving postpartum lactation training increase the level of exclusive breastfeeding.

Keywords Exclusive Breastfeeding; Human Milk; Child Health
*Infertility Reproductive Health Research Center, Shahid Beheshti Medical University, Tehran, Iran ${ }^{1}$ Nursing \& Midwifery Faculty, Shahid Beheshti Medical University, Tehran, Iran

2Paramedical Faculty, Shahid Beheshti Medical University, Tehran, Iran

\section{Correspondence}

Address: -

Phone: -

Fax: -

khodakarami@yahoo.com

\section{Article History}

Received: February 14, 2016

Accepted: May 21, 2016

ePublished: June 15, 2017

\section{I T A T I O N L I N KS}

[1] Maternal and child health [2] Global strategy on infant and young child feeding [3] Golden key for health: Exclusive feeding with breast milk [4] Monitoring and evaluation of reproductive health [5] Prevalence of exclusive breast feeding and its determiners in the first 3 months of life in the south of Brazil [6] Factors associated with exclusive breast feeding of infants in Poland [7] Factors associated with exclusive breastfeeding in Accra, Ghana [8] Studies on exclusive breast feeding; A report on associated factors in an Africa population [9] Breast feeding initiation and determinants of exclusive breast feeding a questionnaire survey in urban population of western Nepal [10] Breast-feeding initiation and exclusive breast-feeding in rural Vietnam [11] Comparison of the breastfeeding patterns of mothers who delivered their babies pervagina and via cesarean section: An observational study using the LATCH breastfeeding charting system [12] Effect of delivery method and timing of breastfeeding initiation on breastfeeding outcomes in Taiwan [13] A Survey on the Pattern of Mothers' Breastfeeding in Babol [14] Barriers to timely initiation of breastfeeding among mothers of healthy full-term babies who deliver at the University of Port Harcourt Teaching Hospital [15] Factors associated with exclusive breast feeding in Japan for activities to support child rearing with breast feeding [16] Comparative study on the lactation pattern of mothers of 11-12 months old children and daughters referring to Semnan Medical Sciences Centers [17] Breast Feeding at 6 weeks and predictive factors [18] Factors adversely associated with breast feeding in Newzealand [19] Prevalence of exclusive breastfeeding and its related factors in infants in Zahedan in 2000-2001 [20] The reasons for failure of exclusive breast-feeding in health centers in Gorgan (1999-2000) [21] Factors associated with exclusive breast feeding in children under four months old in Botu Catu-Sp, Brazil 
شاخص توفيق برنامههاى شير مادر در دنيا، آمار تغذيه انحصارى با مارديا

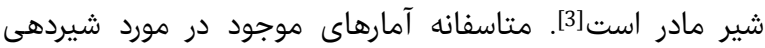

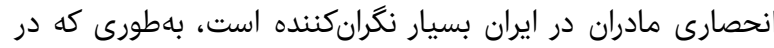

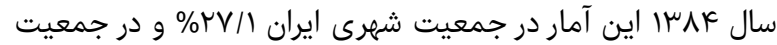

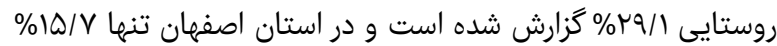

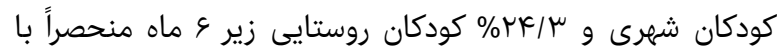
شير مادر تغذيه شدهاند [4].].

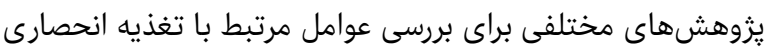

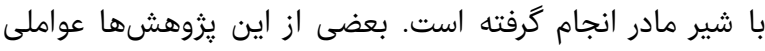

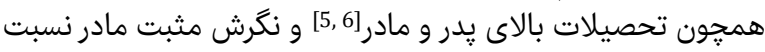

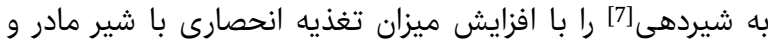

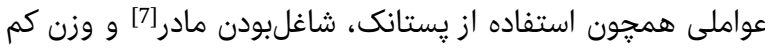

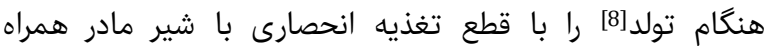

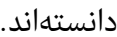

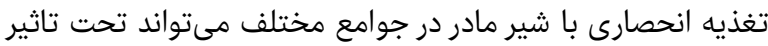

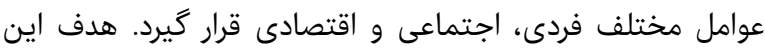

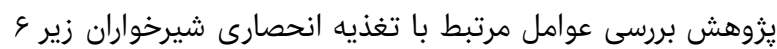

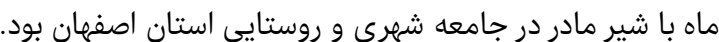

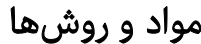

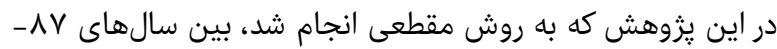

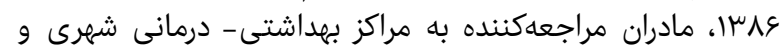

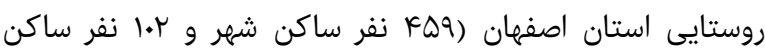

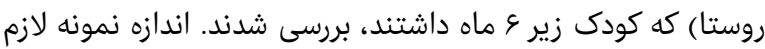

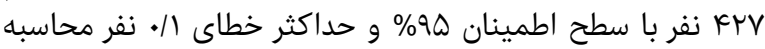

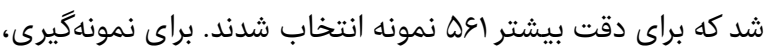

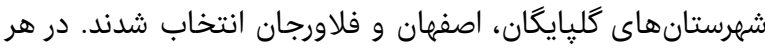

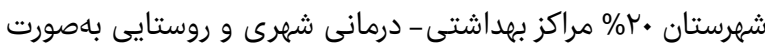

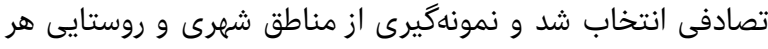

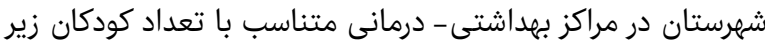

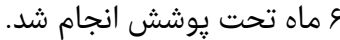

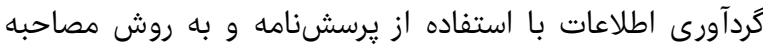

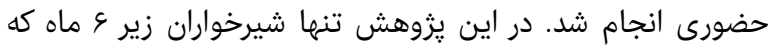

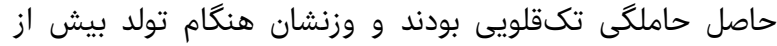

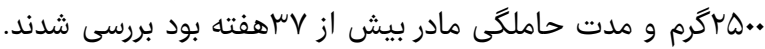

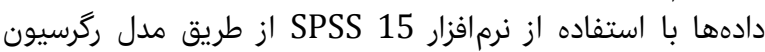

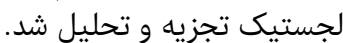

يافتهها

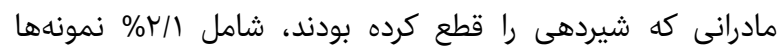

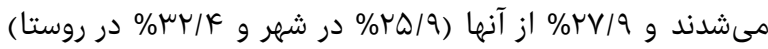

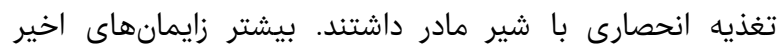

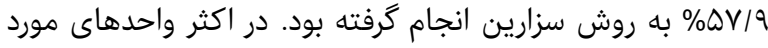

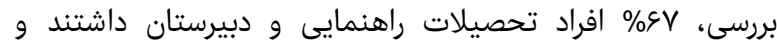

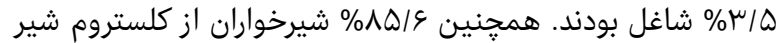

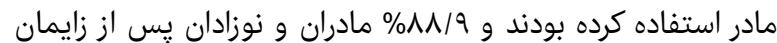

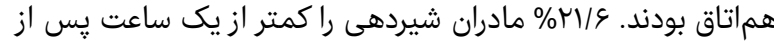

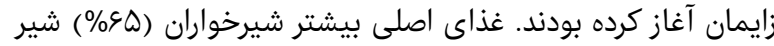

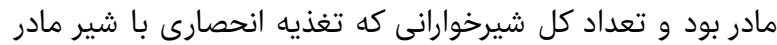

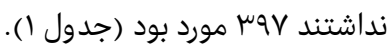

بررسى عوامل مرتبط تغذيه انحصارى با شير مادر

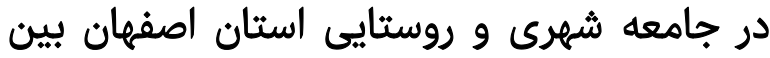

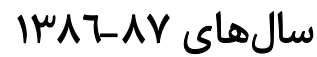
دانشكده يرستارى و مامايى، دانشكاه علوم يزشكى شهريد

MSc "ناهيد خداكرمى

مركز تحقيقات بهداشت بارئ بارورى و نابارورى، دانشكاه علوم يزشكى شهيد بهشتى،

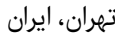

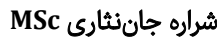

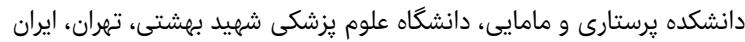

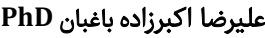
دانشكده يِيرايزشكى، دانشكاه علوم يزشكى شهيد بهشئى، تهران، ايران

جكيده

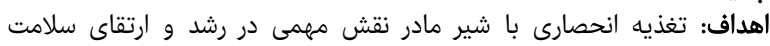

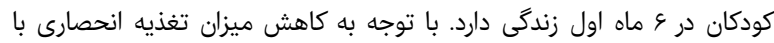

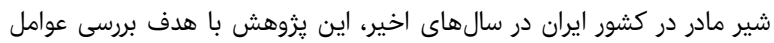

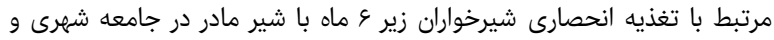
روستايى استان اصفهان انجام شد.

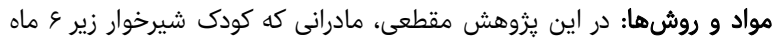

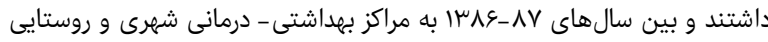

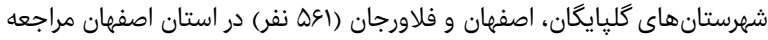

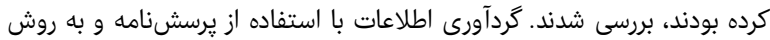

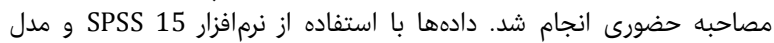

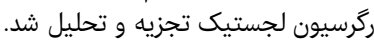

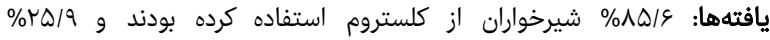

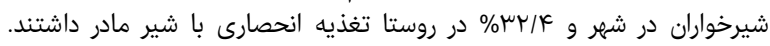

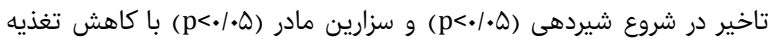

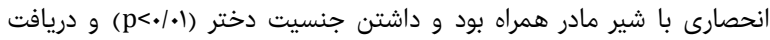

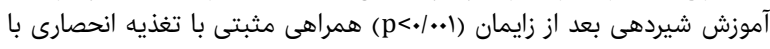

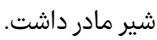
نتيجهيرى: تاخير در شروع شيردهى و سزارين مادر مادر از عوامل كاهش سطح

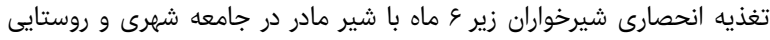

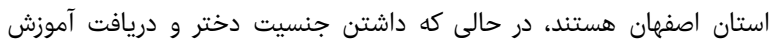

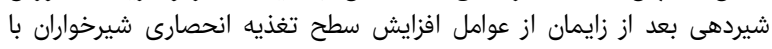
شير مادر هستند. كليدوارهها: تغذيه انحصارى، شير مادر، سلامت كودكان

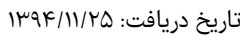

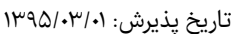
"نويسنده مسئول: khodakarami@yahoo.com

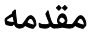

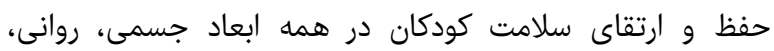

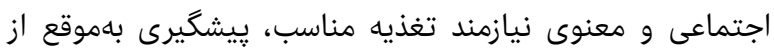

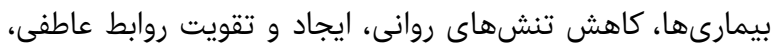

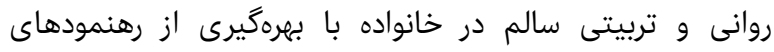

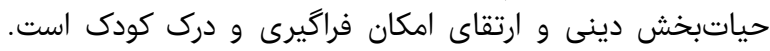

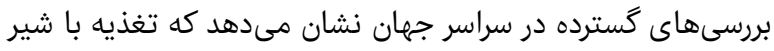

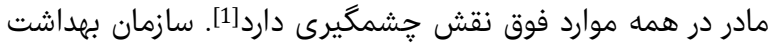

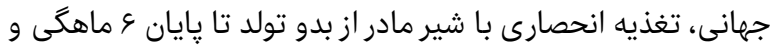

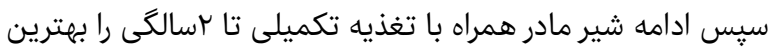

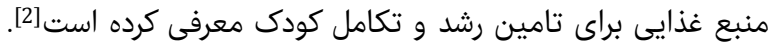
با توجه به مزاياى تغذيه انحصارى با شير ماديد مادر، مهمترين 
بررسى عوامل مرتبط تغذيه انحصارى با شير مادر در جامعه شهرى و روستايى استان اصفهان بين ... الهاديه

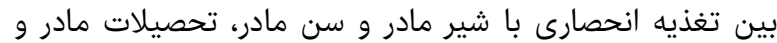

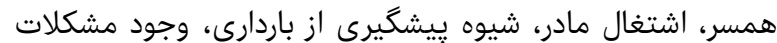

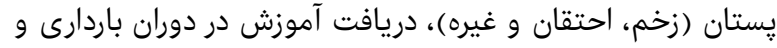

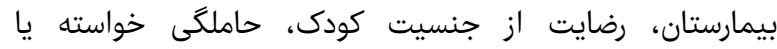

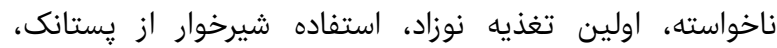

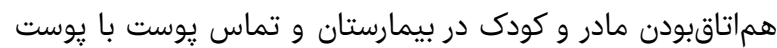

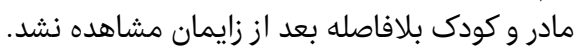

جدول F) نتايج نهايى ارزيابى عوامل مرتبط با تغذيه انحصارى با شير مادر در

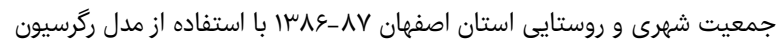

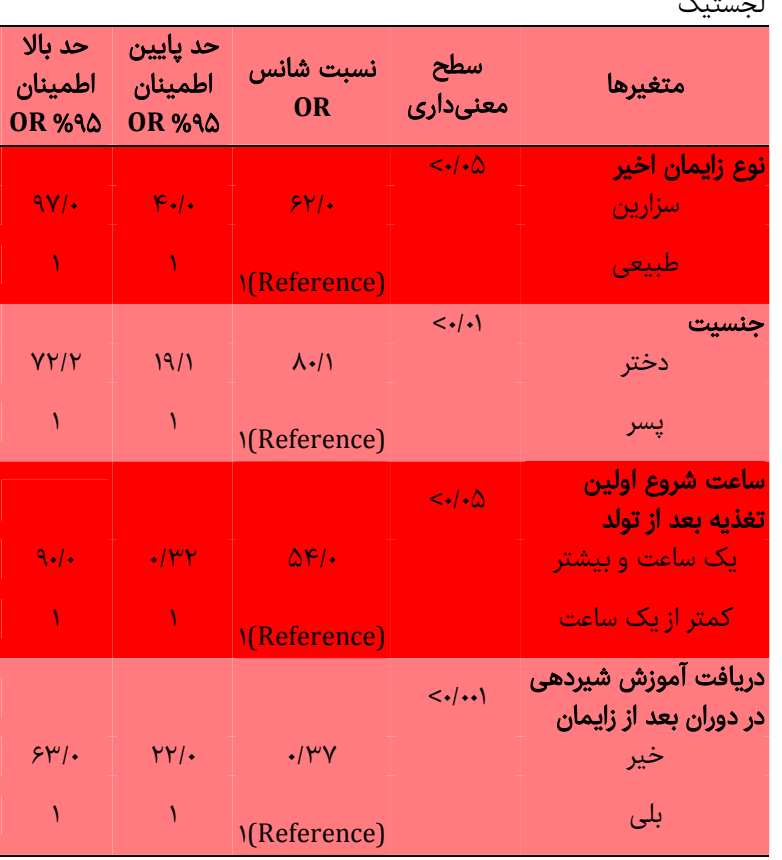

بحث

هدف يزوهش بروسى عوامل مرتبط با تغذيه انحصارى شيرخواران

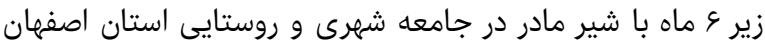

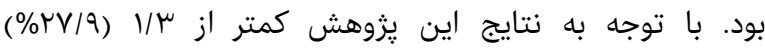

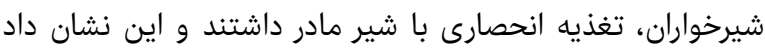

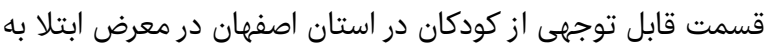

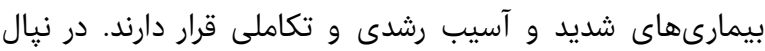

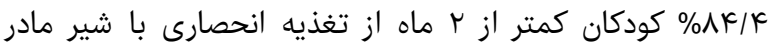

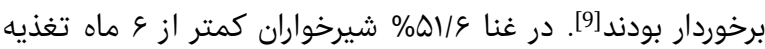

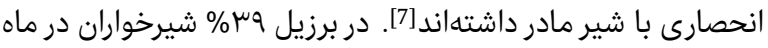

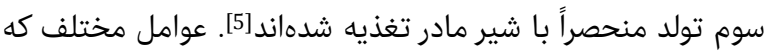

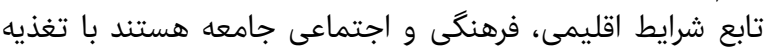

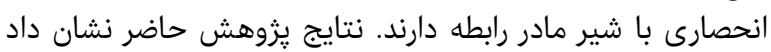

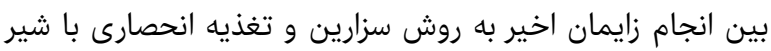

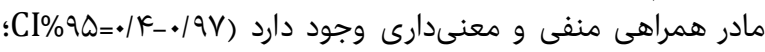

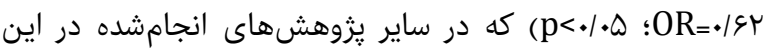

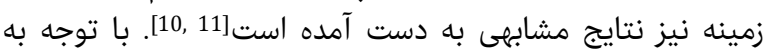

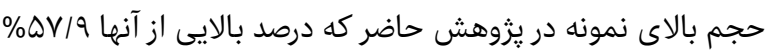

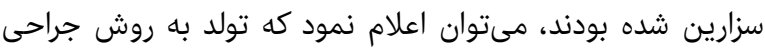

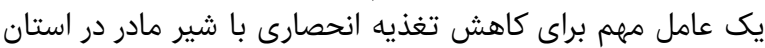

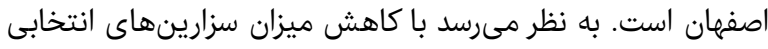

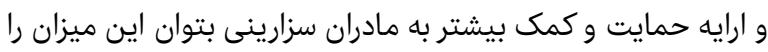

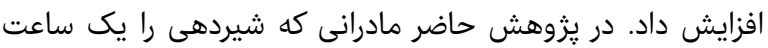

آب در بين مواد مصرفى شيرخواران كمتر از ع ماه بيشتر از ساير

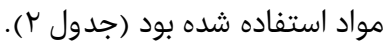

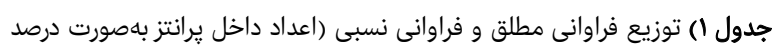

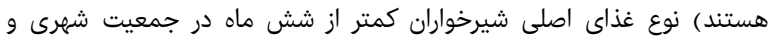

\begin{tabular}{|c|c|}
\hline 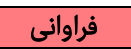 & نوع غذاى اصلى شيرخوار \\
\hline$r \Delta \Lambda(\varphi \Delta / \cdot)$ & شير مادر \\
\hline$\Delta Q(1 \% / Q)$ & شير مادر به علاوه شير خشك \\
\hline $\mathrm{V9}(19 / 9)$ & شير مادر به علاوه غذاى كمكى \\
\hline$\Delta(1 / r)$ & شير مادر به علاوه شير خشك به همراه غذاى كمكى \\
\hline
\end{tabular}

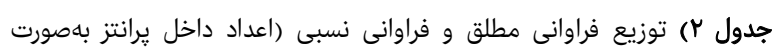

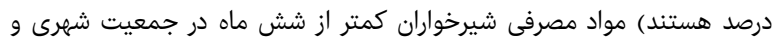

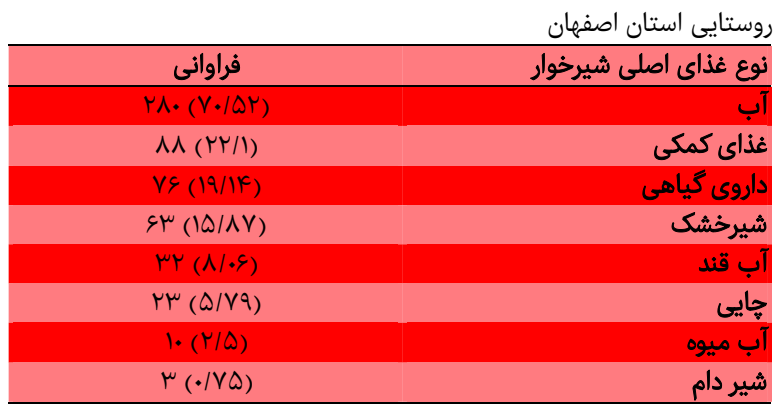

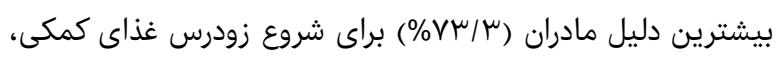

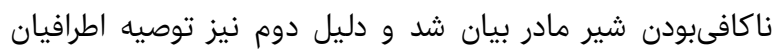

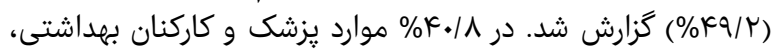

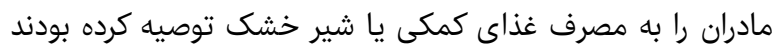

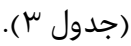

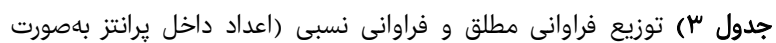

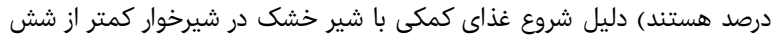

\begin{tabular}{|c|c|}
\hline فراوانى & دليل مصرف غذاى كمكى يا شيرخشك \\
\hline $1 . \kappa\left(\mu / V^{\mu}\right)$ & ن اكافىبودن شير مادر \\
\hline $19\left(k / / \mu^{\mu}\right)$ & كريه و بىقرارى كودى \\
\hline$F(\Lambda / T)$ & شاغلبودن مادر \\
\hline$r(r / I)$ & بيمارى مادر \\
\hline \multirow[t]{2}{*}{$r(r / 1)$} & مصرف دارو توسط مادر \\
\hline & توصيه اطرافيان \\
\hline$\kappa \wedge(\Lambda / \mu \mu)$ & 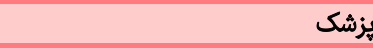 \\
\hline$k \cdot(V / \cdot)$ & كاركنان بهداشتى \\
\hline$\Delta(\Delta / \mu)$ & 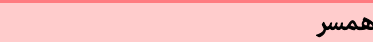 \\
\hline$\Delta(\Delta / \mu)$ & 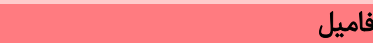 \\
\hline$r(k / 1)$ & 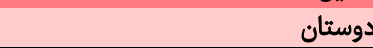 \\
\hline
\end{tabular}

بيشترين دليل مصرف آب (هڤه\%) توصيه اطرافيان بود كه در

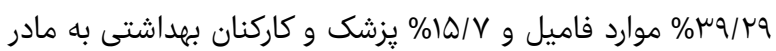

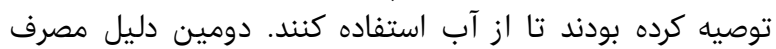

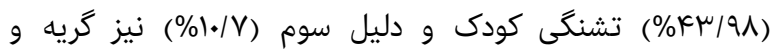

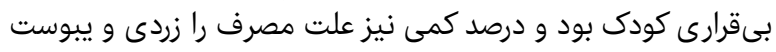
كودى گزارش كردند.

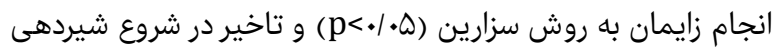

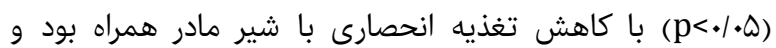

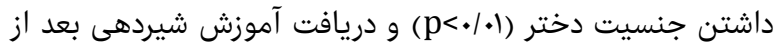

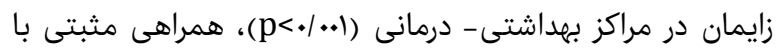

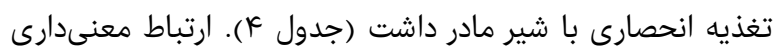

Volume 2, Issue 1, Spring 2017 
تشكر و قدردانى: موردى از سوى نويسندكان ذكر نشده است.

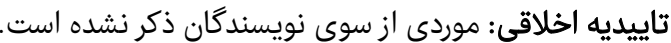

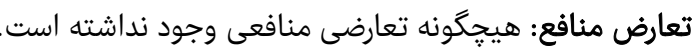

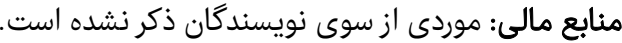

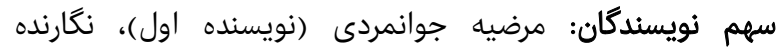

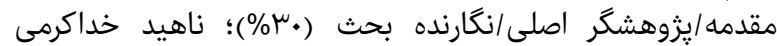

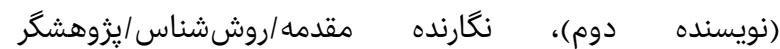

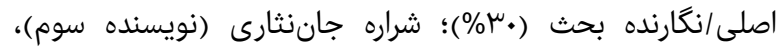

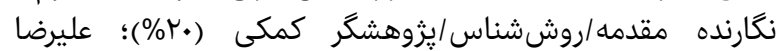

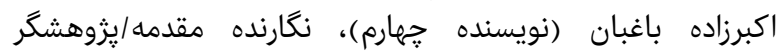

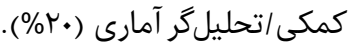

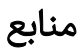

1- Soltani R, Parsayi P. Maternal and child health. 2th ed. Tehran: Sanjesh publication. 2005. [Persian]

2- World Health Organization, UNICEF. global strategy on infant and young child feeding [Internet]. Geneva: World Health Organization; 2003 [cited 2002 jul 2]. Avilable from:

http://www.who.int/nutrition/publications/infantfeedi ng/9241562218/en/.

3- Bahrami M. Golden key for health: Exclusive feeding with breast milk. Shir e-Mathar. 2004;5(18):4-7. [Persian]

4- Ministry of Health and Medical Education of Iran. Monitoring and evaluation of reproductive health Tehran: Ministry of Health and Medical Education of Iran; 2005. [Persian]

5- Mascarenhas ML, Albernaz EP, Silva MB, Silveria MB. Prevalence of exclusive breast feeding and its determiners in the first 3 months of life in the south of Brazil. J Pediatr. 2006;2(4):289-94.

6- Wojdon-Godak E, Mikiel-Kostyra K, Mazur J. factors associated with exclusive breast feeding of infants in Poland. Med Wieku Rozwoi. 2000;4(3 suppl 1):15-25. [Polish]

7- Aidam BA, Perz-Escamilla R, Lartey A, Aidam J. Factors associated with exclusive breastfeeding in Accra, Ghana. Eur J Clin Nutr. 2005;59(6):789-96.

8- Eregie Co . Studies on exclusive breast feeding; A report on associated factors in an Africa population. J Trop pediatr. 1998 ;44(3):172-3.

9- Chanrashekhar TS, Joshi HS, binu VS, Shankar PR, Rana MS, Ramachandran U. Breast feeding initiation and determinants of exclusive breast feeding a questionnaire survey in urban population of western Nepal. Public Health Nutr. 2007;10(2):192-7.

10- Duong DV, Binns CW, Lee AH. Breast-feeding initiation and exclusive breast-feeding in rural Vietnam. Public Health Nutr. 2004;7(6):795-9.

11- Cakmak H, Kuguoglu S. Comparison of the breastfeeding patterns of mothers who delivered their babies pervagina and via cesarean section: An observational study using the LATCH breastfeeding charting system. Int J Nurs Stud. 2007;44(7):1128-37.

12- Tai CJ, Chien LY. Effect of delivery method and timing of breastfeeding initiation on breastfeeding outcomes in Taiwan. Birth. 2007;34(2):123-30.

13- Hajiyan KA. A Survey on the Pattern of Mothers' Breastfeeding in Babol. Sch Med Shahid Beheshti Univ Med Sci. 2001;25(4):205-11. بعد از زايمان شروع كرده بودند، تغذيه انحصارى در آنها بهاطور

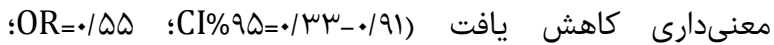

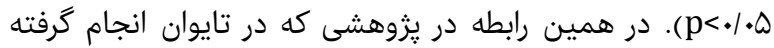

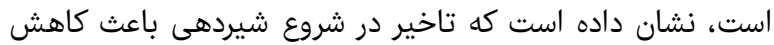

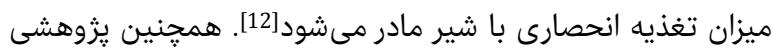

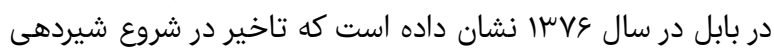

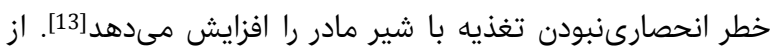

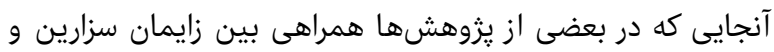

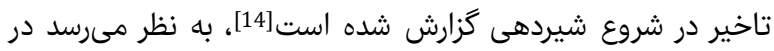

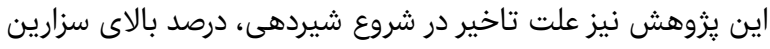

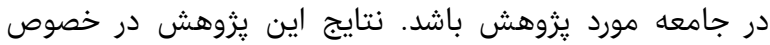

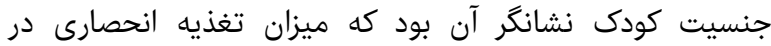

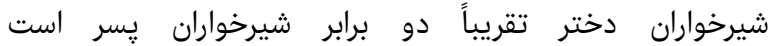

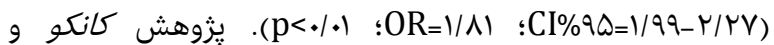

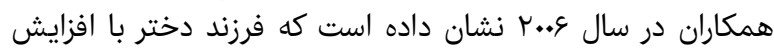

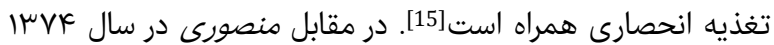

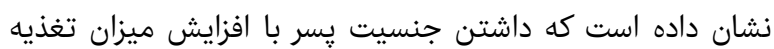

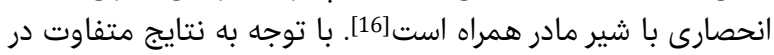

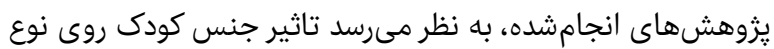

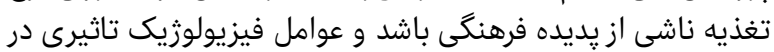

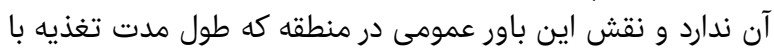

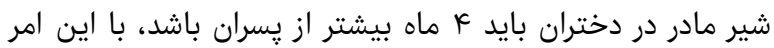

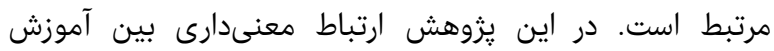

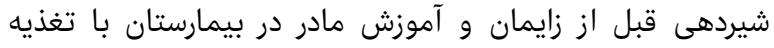

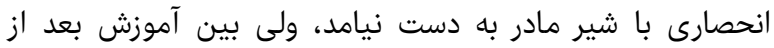

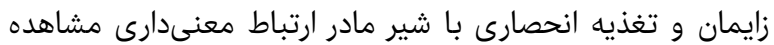

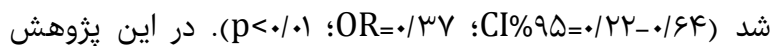

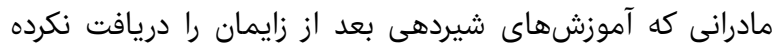

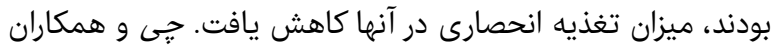

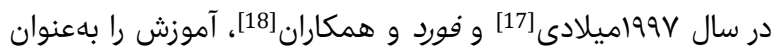

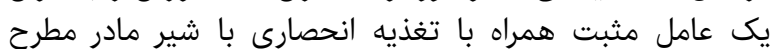

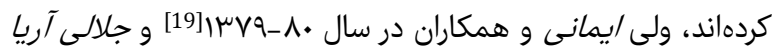

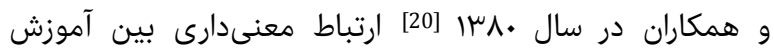

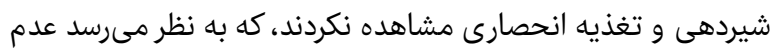

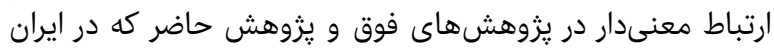

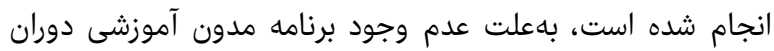

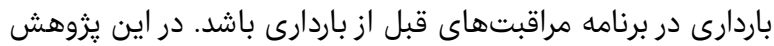

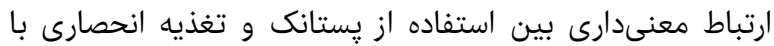

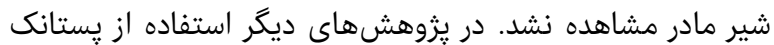

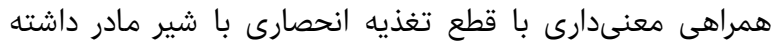

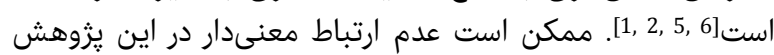

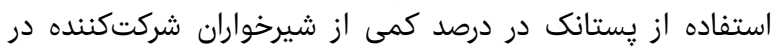

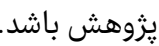

\section{نتيجه}

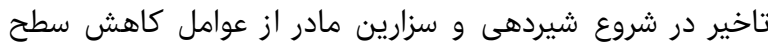

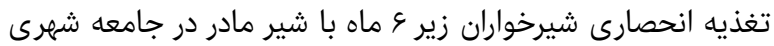

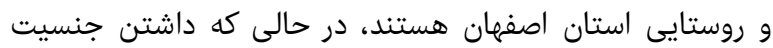

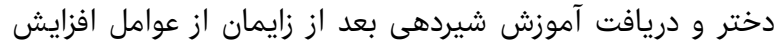

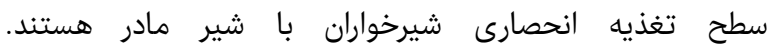


بررسى عوامل مرتبط تغذيه انحصارى با شير مادر در جامعه شهرى و روستايى استان اصفهان بين ... سه

18- Ford RPK, Mitchhell EA, Scrag R, stewart AW, Taylor

BJ, Allen EM. Factors adversely associated with breast feeding in Newzealand. J pediatr Child Health. 1994;30(6):483-6.

19-Imani M, Mohammadi M, Rakhshani F, Shafiei S. Prevalence of exclusive breastfeeding and its related factors in infants in Zahedan in 2000-2001. Feyz sci res J. 2003;7(2):26-33.

20- Jalaly Aria KJ, Sanagoo A, Jooybari L. The reasons for failure of exclusive breast-feeding in health centers in Gorgan (1999-2000). J Gorgan Univ Med Sci. 2001;3(2):81-6.

21- de Barros Leite Carvalhaes MA, de Lima Parada CM, da Costa MP. factors associated with exclusive breast feeding in children under four months old in Botu CatuSp, Brazil. Rev Lat Am Enfermagem. 2007;15(1):62-9.
14- Awi DD, Alikor EA. Barriers to timely initiation of breastfeeding among mothers of healthy full-term babies who deliver at the University of Port Harcourt Teaching Hospital. Niger J Clin Pract. 2006;9(1):57-64.

15- Kaneko A, Kaneita Y, Yokoyama E, Miyake T, Harano $\mathrm{S}$, Suzuki K, etal . Factors associated with exclusive breast feeding in Japan for activities to support child rearing with breast feeding. J Epidemiol. 2006;16(2):57-63.

16- Mansouri A, Dabaghi A, Mehri F, Haghani $H$. Comparative study on the lactation pattern of mothers of 11-12 months old children and daughters referring to Semnan Medical Sciences Centers [Dissertation]. Tehran: Tehran Medical Universisty; 1996. [Persian] 17- Chye JK ,Zain Z, Lim WL ,Lim CT. Breast Feeding at 6 weeks and predictive factors. J Trop pediatr. 1997;43(5):284-92. 